\title{
Modélisation et expérimentation du décapage par jet d'eau haute pression
}

\author{
Tarek Mabrouki ${ }^{1, a}$, Alain Cornier ${ }^{2}$, Hafiz Osman $^{3}$ et Kadour Raissi ${ }^{4}$ \\ 1 INSA-Lyon, Laboratoire LaMCoS, Bât. Joseph Jacquard, 27 avenue Jean Capelle, 69621 Villeurbanne Cedex, France \\ 2 ENSAM Institut Conception, Mécanique et Environnement, Savoie Technolac-BP 295, 73375 Le Bourget Du Lac Cedex, \\ France \\ 3 Valeo Transmissions - R\&D Centre, ZI Nord, Rue de poulainville, 80009 Amiens Cedex 1, France \\ 4 ENSAM Paris, 151 Bd de l'hôpital, 75013 Paris, France
}

Reçu le 20 février 2002, accepté le 5 janvier 2003

\begin{abstract}
Résumé - Cet article propose une modélisation numérique tridimensionnelle du procédé de décapage par jet d'eau HP. L'exemple traité concerne un dépôt aéronautique : peinture de type polyuréthane déposée sur un métal homogène infini, A2024, de type carlingue d'avion. Le calcul numérique a été effectué en utilisant le code DYNA3D. Les résultats hydrodynamiques montrent l'importance de l'aplatissement du jet sur la cible traitée. Il s'avère qu'un jet d'eau en mouvement par rapport à la cible à décaper génère des contraintes de cisaillement provoquant un enlèvement du revêtement selon un mode d'érosion. En terme de modélisation, ce phénomène a été mis en évidence grâce à un critère seuil qui simule l'enlèvement des mailles lagrangiennes ainsi soumises aux contraintes générées par l'action du jet sur la cible. Cette érosion est accentuée par un autre phénomène qui se traduit par une concentration des contraintes de traction sur la cible selon la ligne médiane de l'empreinte, lieu géométrique du déplacement du centre du jet. Ces contraintes peuvent générer des fissures qui à leur tour peuvent être des sites privilégiés traversés par des micro-jets à vitesses élevées. L'enlèvement du revêtement est ainsi accéléré. Les résultats ont été validés par des essais expérimentaux.
\end{abstract}

Mots clés : jet d'eau / décapage / érosion / modélisation

Abstract - Modelling and experimentation of stripping by high-pressure waterjet. This paper deals with the 3D-numerical modelling of decoating process by a moving waterjet tool. The example of aeronautic coating (polyurethane) deposit on an infinite homogenous metal (A2024) was treated (case of plane keelson). The numerical computation was carried out using DYNA3D code. Hydrodynamic results show the importance of the jet flattening on the treated target. It appears clearly that moving watertjet introduces during decoating process shear stresses causing coating removal by erosion mode. The latter has been simulated thanks to a failure criterion of Lagrangian elements illustrating the coating removal under high velocity moving waterjet. This erosion is accentuated by waterjet stretching effects, which cause tearing of the coating. Consequently, discontinuous cracks at the median line of the coating imprint can be observed. These cracks can be privileged sites, which are traversed by speedy micro-jets accelerating the coating removal. The results were demonstrated by experimental tests.

Key words: waterjet / stripping / erosion / modelling

\section{Introduction}

L'industrie de préparation de surface a besoin de nouvelles technologies pour les substituer aux procédés de décapage existants. En effet, les méthodes actuelles sont souvent trop coûteuses et limitées par des règles environnementales. L'application des protocoles de Montréal et

a Auteur correspondant : Tarek.Mabrouki@insa-lyon.fr de Kyoto conduit à la disparition de solvants de décapage (composés organo-volatiles), certes polluants, mais très efficaces d'un point de vue industriel. Par ailleurs, l'utilisation d'un procédé de projection d'abrasif tel que le sablage, est aussi interdite en raison des conséquences environnementales liées à la dispersion dans l'air ou l'eau de métaux lourds utilisés (décapage par sablage des revêtements marins : cuivre, cadmium et plomb). 
Parmi les nouvelles technologies qui semblent être capables de résoudre les problèmes actuels inhérents au décapage, nous notons l'usage du jet d'eau haute pression (HP) qui a été utilisé pendant longtemps pour des opérations de nettoyage (turbine à gaz, pièces de moteur, échangeurs de chaleur, etc.) et de découpage (métaux, plastique, béton).

Depuis peu, les secteurs aéronautiques et navals emploient ce procédé pour effectuer des opérations de remise en état des réacteurs (enlèvement des résidus de combustion) et de la coque des navires. Des concepteurs recommandent de traiter par projection de jet d'eau HP les opérations de nettoyage et de décapage des dépôts techniques, tels que les revêtements de type barrière thermique. Bien que ce procédé bénéficie d'améliorations en terme d'automatisation et de développement des outils de décapage, il présente des inconvénients : problèmes de nuisance acoustique, de mauvaise répartition énergétique des jets, etc. Par conséquent, en plus des études paramétriques du procédé qui apparaissent indispensables, des études concernant l'interaction des jets avec les matières à décaper sont un outil puissant et efficace pour développer le procédé. Un de ces problèmes peut être remarqué lors du décapage avec des têtes dites rotatives (Fig. 1a). En effet, dans beaucoup de cas, la composition de mouvement de rotation de ces têtes avec la vitesse d'avance, génère une mauvaise uniformité au niveau de la zone décapée, une mauvaise sélectivité des couches et une non-maîtrise de l'intégrité de la surface ainsi traitée.

Afin d'améliorer ce procédé, plusieurs auteurs ont publié leurs observations au sujet de l'interaction jet-cible. Cependant, la plupart de ces publications sont d'ordre expérimental [1-4] et chaque cas d'étude correspond à des conditions d'expérimentation spécifiques difficilement reproductibles. En outre, quelques phénomènes impliqués d cette interaction restent encore jusqu'ici peu expliqués. A ce jour, il existe très peu de publications $[5,6]$ traitant de la modélisation du décapage par jet d'eau HP, à part les modèles de Leu et al. [5] et de Meng et al. [6] très récents. Ces auteurs ont développé deux modèles mathématiques traitant du nettoyage par et ceci pour deux configurations cinématiques du jet, respectivement, stationnaire et en mouvement par rapport à la cible à traiter. Les modèles sont établis en supposant que le jet d'eau est un ensemble de gouttelettes et en considérant que le nettoyage se produit quand la contrainte dynamique équivalente, due à l'action des gouttelettes de l'eau sur la cible, est supérieure ou égale à la contrainte limite de la rupture du matériau heurté [7]. Toutefois, dans leur formulation du problème, ces auteurs supposent que le décapage est réalisé d'une façon complète dès qu'un certain seuil de rupture est atteint. Par conséquent, l'importance de l'évolution du décapage en fonction du temps d'exposition de la pièce au jet est négligée. En outre, les caractéristiques d'interface revêtement-substrat ne sont pas prises en considération. La démarche de ces auteurs est, principalement, focalisée sur l'étude de l'influence de certains paramètres de travail sur la largeur du nettoyage. Cela, bien sûr, ne réduit pas la qualité de ces travaux qui représentent de très bonnes références concernant la modélisation du nettoyage.

Le présent travail, consiste à étudier les mécanismes qui interviennent lors de l'interaction d'un jet avec un alliage d'aluminium (A2024) revêtu par une couche de polyuréthane. Nous insistons sur le fait que le développement du procédé de décapage, en terme de conception d'une génération d'outils performants ou optimisation d'outils existants, peut être réalisé par la compréhension de cette interaction jet-cible. L'étude de l'interaction d'un jet immobile avec ce même type de cible a été traitée dans des publications précédentes [8-12].

\section{Données de l'étude}

Une opération de décapage consiste à enlever d'un substrat un matériau inutilisable (cas du nettoyage) ou endommagé, en vue de réaliser une opération de restauration. Logiquement le décapage doit se réaliser sans attaquer ni changer la structure du substrat tout en assurant des critères de qualité (sélectivité, uniformité, etc.). Cette vision nous amène à présenter les paramètres influençant l'évolution du décapage par jet d'eau HP. Ces paramètres peuvent être répartis en trois grandes familles (Fig. 1b) :

- paramètres de la tête de décapage : ils englobent la géométrie de la buse, la pression et le débit d'eau;

- paramètres du jet : ils concernent la formation du jet dans l'air : l'évasement du jet, sa composition, sa cinétique, son inclinaison par rapport à la cible, la distance de tir du jet et ses propriétés physiques (masse volumique, viscosité) ;

- paramètres de la cible : ils englobent les caractéristiques mécaniques intrinsèques, les propriétés physico-chimiques des matériaux (dépôt + substrat) ainsi que les paramètres géométriques de la cible.

Ces différents paramètres interagissent et constituent un ensemble complexe quant au décapage par jet d'eau.

En décapage industriel, les outils utilisés sont des têtes multijets rotatives munies de plusieurs buses cylindriques disposées sur des diamètres différents de façon à uniformiser la répartition de l'énergie cinétique des jets en composant le mouvement de rotation de la tête avec son mouvement d'avance (Fig. 1a) : l'enlèvement de matière est réalisé par un mouvement cycloïdal de chaque jet heurtant la cible (Fig. 2). Le tableau 1 donne les paramètres de fonctionnement d'une tête rotative multibuses (Fig. 1a).

L'étude consiste à analyser l'interaction d'un seul jet avec une cible revêtue. Le cas traité concerne le décapage d'un revêtement polyuréthane $(100 \mu \mathrm{m}$, peinture de type carlingue d'avion) déposé sur une plaque d'alliage d'aluminium supposée infinie et d'épaisseur est égale à 1 millimètre (A2024).

Les vitesses utilisées en décapage varient, généralement, entre $200 \mathrm{~m} / \mathrm{s}$ et $900 \mathrm{~m} / \mathrm{s}$. Les distances de tir dépendent, dans des opérations de décapage manuel, de l'habileté de l'opérateur. Dans le cas des opérations de décapage robotisées et destinées au traitement des pièces onéreuses (carlingue d'avion, carrosserie 


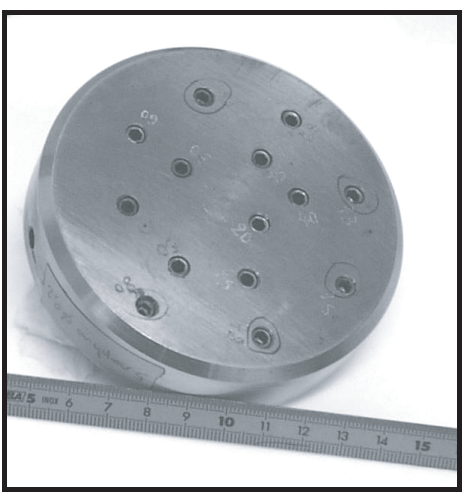

(a)

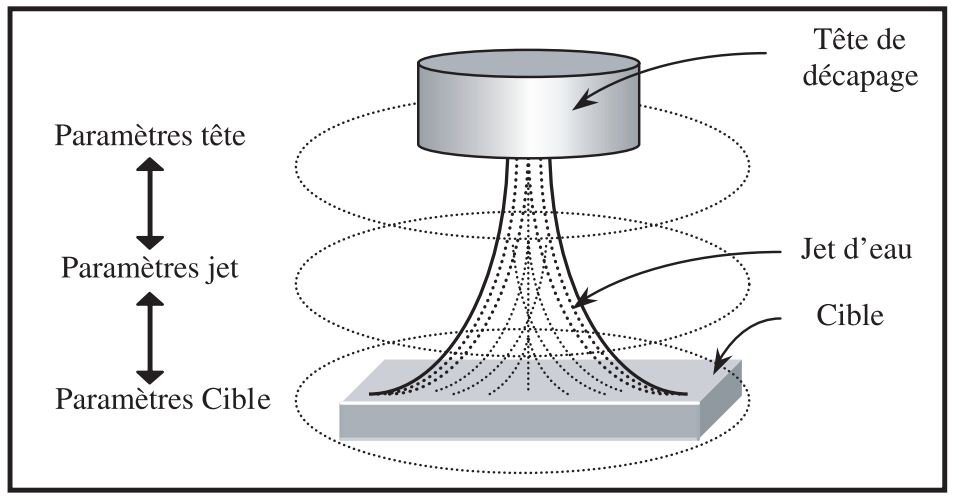

(b)

Fig. 1. (a) Tête multi-buses de décapage. (b) Paramètres de décapage.

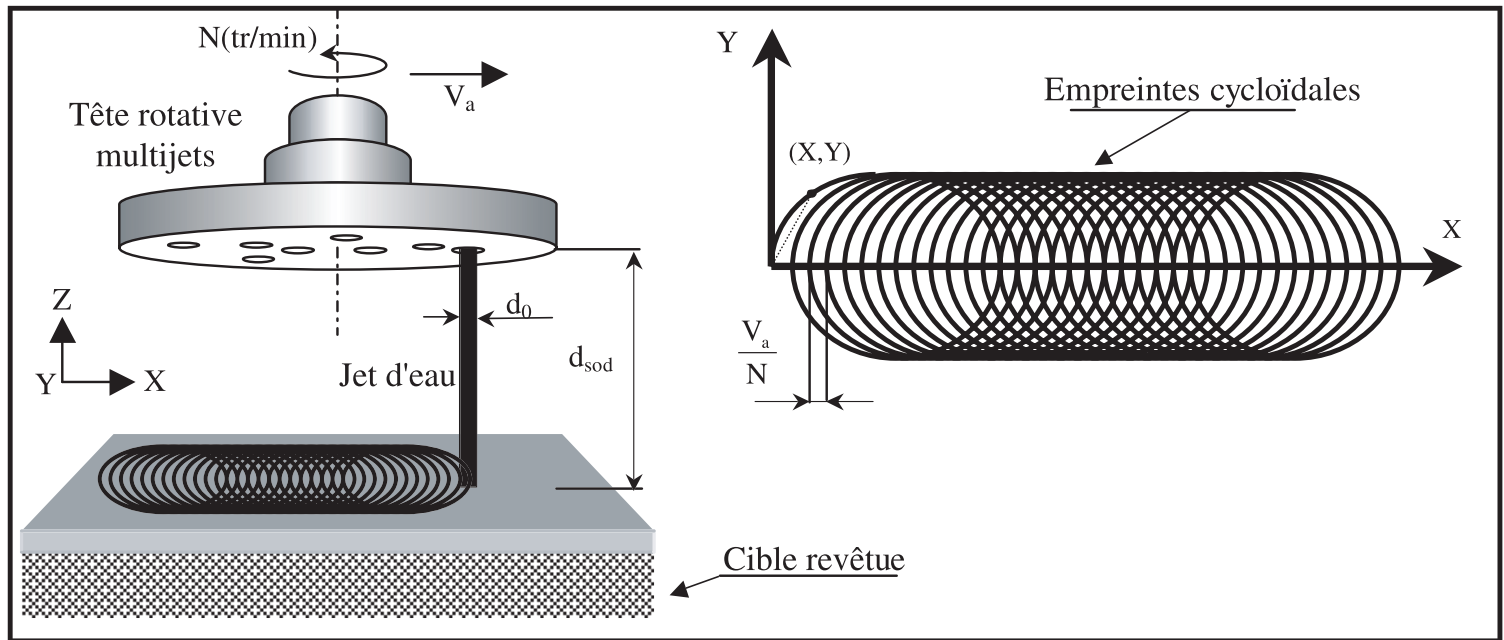

Fig. 2. Représentation schématique de l'action d'un jet d'eau HP issu d'une tête rotative monojet, sur une cible revêtue.

Tableau 1. Paramètres de fonctionnement d'une tête rotative.

\begin{tabular}{ccccccc}
\hline Pression & Diamètre buse & Nombre buse & $\begin{array}{c}\text { Vitesse rotation } \\
N(\mathrm{tr} / \mathrm{min})\end{array}$ & $\begin{array}{c}\text { Distance de tir } \\
d_{\text {tir }}(\mathrm{mm})\end{array}$ & $\begin{array}{c}\text { Vitesse avance } \\
V_{a}(\mathrm{~mm} / \mathrm{min})\end{array}$ & $\begin{array}{c}\text { Angle de tir } \\
{ }^{\circ}(\mathrm{deg})\end{array}$ \\
\hline Maxi. 3850 & $0,05 \rightarrow 0,5$ & Maxi. 13 & $40 \rightarrow 1500$ & Sup. à 12 & $50 \rightarrow 8000$ & $0 \rightarrow 45$ \\
\hline
\end{tabular}

automobile, etc.), les distances de tir varient de $12 \mathrm{~mm}$ à $120 \mathrm{~mm}$ pour des diamètres de buse $d_{0}$ variant de $0,05 \mathrm{~mm}$ à $0,5 \mathrm{~mm}$.

Par ailleurs, vu la complexité de la structure d'un jet d'eau, que ce soit du point de vue phasique ou cinétique, nous nous contentons de traiter seulement l'action de la zone initiale du jet sur la cible (Fig. 3). Le choix de la longueur de cette zone se réfère aux travaux de Yanaida [13], Neusen et al. [14] et Tikhomirov et al. [15]. Notons que la longueur adimensionnelle $\frac{x_{c}}{d_{0}}$ de cette zone correspond à une vitesse uniforme et une structure monophasique, et est telle que $: \frac{x_{\mathrm{c}}}{d_{0}} \in[20,150]$ (Fig. 3). En conséquence, pour une buse de diamètre $d_{0}=0,3 \mathrm{~mm}$ et pour une distance de tir telle que : $d_{\mathrm{tir}}=30 \mathrm{~mm}$, la valeur du rapport $\frac{x_{\mathrm{c}}}{d_{0}}$ vaut 100 . Une distance de tir de $30 \mathrm{~mm}$ correspond donc à une section cohérente du jet. Cette distance confère ainsi au jet une structure fluidique plutôt monophasique.

\section{Simulation numérique}

Nous signalons que la variation de la distance de tir lors d'un impact du fluide modélisé par le code de calcul LS-DYNA n'a pas d'effet significatif sur l'interaction du jet avec la cible. En effet, ce code ne contient pas de modèle fluidique permettant de simuler la turbulence du jet et son évasement : la problématique traitée consiste à injecter une masse fluidique cylindrique (colonne d'eau) avec une vitesse de $500 \mathrm{~m} / \mathrm{s}$ dans un milieu ambiant (air). Cette masse liquide parcourt une distance avant d'attaquer la cible. Puisque la structure fluidique du jet ne change pas avec cette distance, il est d'intérêt à la minimiser jusqu'à une valeur à partir de laquelle l'interaction est bien mise en évidence (par exemple : possibilité de visualiser et étudier l'aplatissement du jet sur la cible). Cette démarche permet d'assurer un gain de temps de calcul (CPU). 


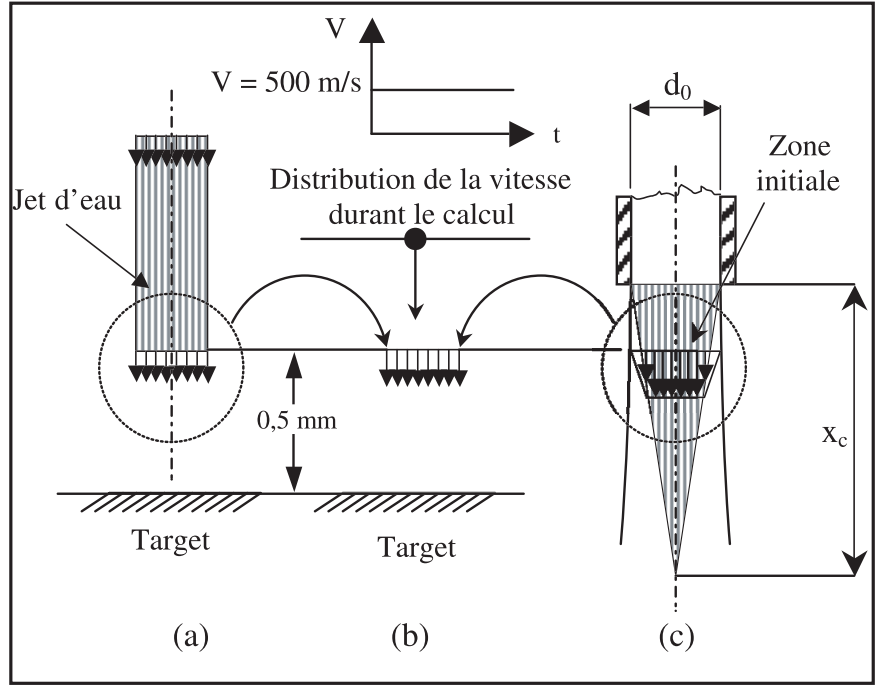

Fig. 3. (a) Jet cylindrique : cas du modèle numérique. (b) Vitesse introduite dans la modélisation. (c) Structure d'un jet accéléré : cas réel.

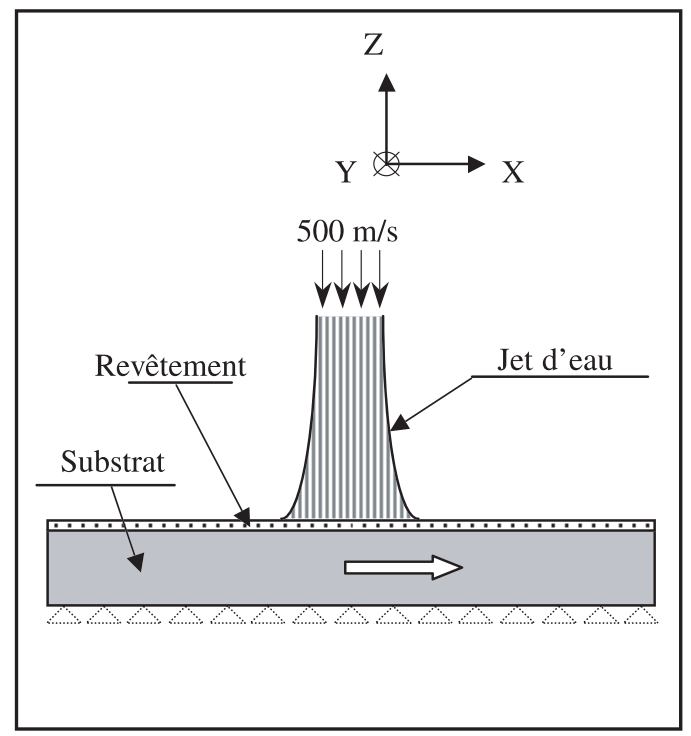

Fig. 4. Représentation du décapage par un jet unique.

Grâce aux données précédentes la distance séparant la cible et la charge hydrodynamique est fixée, au cours de ce calcul numérique, à $0,5 \mathrm{~mm}$. La vitesse de la charge est donc fixée à $500 \mathrm{~m} / \mathrm{s}$ et la section du jet à $d_{0}=0,3 \mathrm{~mm}$. Ainsi, la configuration correspondant au modèle est la simulation d'un jet d'eau mobile (en rotation) heurtant une cible revêtue. Afin de mettre en évidence cette interaction, il est plus facile, d'un point de vue numérique, de donner le mouvement à la cible qu'au jet (Figs. 4 et 5). Cette idée sera explicitée dans ce qui suit.

\subsection{Choix du code de calcul}

Au cours d'une opération de décapage par jet d'eau $\mathrm{HP}$, les structures traitées sont le siège d'effets dynamiques et font apparaître des problèmes de non-linéarité : grandes vitesses de déformations, grandes transformations. Pour cela, nous avons eu recours à un code de calcul qui tient compte de ces problèmes et, qui de plus, est capable de traiter le couplage fluide-structure. Pour ce faire, nous avons utilisé le code de calcul LS-DYNA [16]. Une des premières utilisations de ce code dans le domaine des impacts fluidiques remonte à 1995 avec les travaux d'Adler [17] qui a développé un modèle tridimensionnel traitant l'impact d'une goutte sur une cible déformable. Le but de cette modélisation était d'essayer d'appliquer un code d'éléments finis à un problème d'interaction goutte-cible. Ce code est basé sur un algorithme explicite pour l'intégration du pas de temps et une formulation lagrangienne pour les déplacements. Une formulation eulérienne du mouvement est, aussi, imbriquée dans LS-DYNA. Cette dernière formulation est un cas particulier de la description lagrangienne-eulérienne arbitraire (ALE) [18]. Toutes ces deux dernières formulations sont obtenues en ajoutant des termes de convection à la formulation lagrangienne du mouvement. Ces méthodes sont utiles pour maîtriser les problèmes de distorsion de maillage dans le cas de la description lagrangienne et aussi sont utiles pour décrire les interactions fluide-structure.

La description eulérienne est employée avec la description lagrangienne au moyen d'algorithmes de couplage afin de mettre en exergue l'interaction jet-cible. Il est nécessaire d'indiquer que ce code ne contient pas de modèles traduisant des effets de comportement fluidique turbulent.

Dans la formulation choisie, le comportement du mouvement dans la zone fluidique (jet + air) est décrit par la méthode eulérienne. Par contre, la partie cible (substrat+revêtement) du modèle numérique est traitée en formulation lagrangienne classique. Nous notons, en outre, que cette dernière formulation est toujours la méthode de description du mouvement par défaut dans le code LS-DYNA [16]. Par conséquent, afin d'effectuer un calcul eulérien deux étapes sont nécessaires. La première correspond à une phase lagrangienne pendant laquelle, le maillage enregistre une quantité de mouvement et d'énergie. Les contraintes, l'énergie interne et les forces nodales sont calculées pour chaque élément. Les conditions aux limites et les conditions de chargement sont appliquées comme dans le cas d'un calcul lagrangien conventionnel. La deuxième étape est inhérente à un calcul eulérien. Le maillage déformé lors du calcul lagrangien est ramené à sa position initiale dans le système lagrangien global de référence. La matière est relaxée du maillage. La masse, la quantité de mouvement, l'énergie et les vitesses nodales sont transportées : c'est l'étape d'advection basée sur le schéma de «Donor Cell » associé à l'algorithme HIS (Half Index Shift) corrigé au premier ordre [19].

\subsection{Formulation du problème : Fluide (Euler)-Solide (Lagrange)}

Afin de simuler l'interaction du jet avec la cible, il est indispensable d'effectuer un couplage Euler/Lagrange. Pour ce faire, le maillage lagrangien (zone cible) doit être 


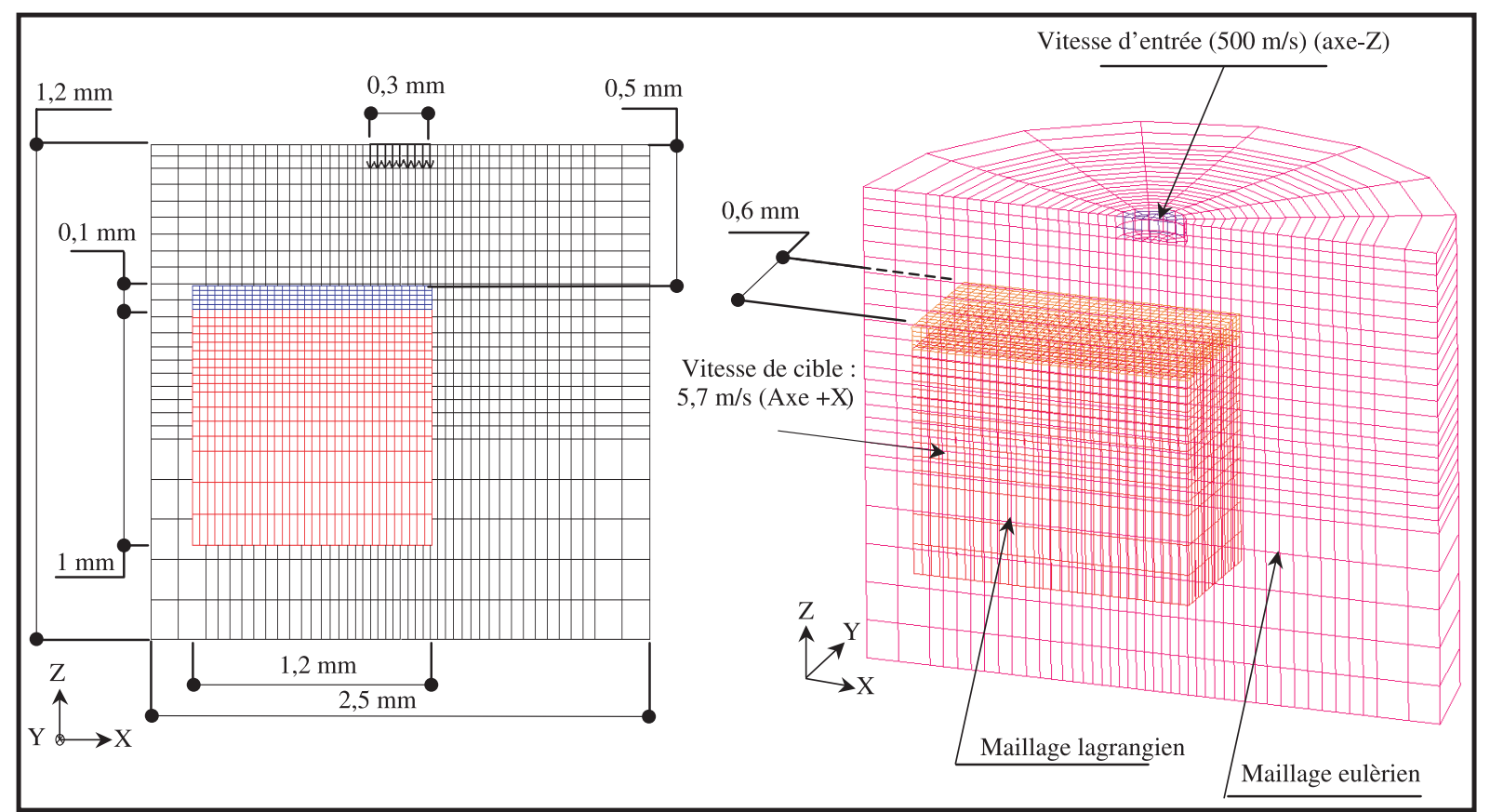

Fig. 5. Modèle : Le maillage lagrangien (polyuréthane+A2024) est immergé dans le maillage eulérien (eau+air). Le nombre d'éléments (éléments brick à 8-nœuds) et de nœuds est, respectivement, 15464 et 21 996. Des conditions de symétrie sont prises en compte (demi-modèle) afin de réduire le temps CPU.

« immergé » dans le maillage eulérien (zone fluide) pour que les structures lagrangiennes puissent interagir avec le maillage eulérien (pas de nouds en communs). Cette dernière approche est différente de l'approche classique relative aux traitements de l'interaction fluide/structure où le couplage est traité par la description d'une surface de contact entre le fluide et la structure. Dans ce cas de figure, une surface de glissement est définie, et les limites géométriques du maillage eulérien, correspondant au contact, deviennent des frontières lagrangiennes. La stratégie générale du couplage Euler-Lagrange, dans le code LS-DYNA, est basée sur la conservation de la quantité de mouvement. Dans ce cas de problème de simulation, un couplage dit « couplage par vitesse » est utilisé. Les accélérations et les vitesses des points lagrangiens de la structure sont couplées aux accélérations et vitesses lagrangiennes du fluide contenu dans les cellules eulériennes.

Il est à noter que le code LS-DYNA comporte un critère d'érosion des mailles lagrangiennes. Il consiste à introduire un seuil de rupture correspondant à un éclatement des éléments hexaédriques lorsqu'ils atteignent leur taux d'allongement à la rupture (érosion numérique) [16]. Ce taux est estimé égal à $21 \%$ de la taille de la maille sans chargement et est déterminé en ajustant les résultats expérimentaux à ceux trouvés numériquement. Nous avons appliqué ce critère au comportement élasto-plastique de la cible et dont la loi d'écrouissage est isotrope.

Les étapes du couplage euler-lagrange sont les suivantes :

- déclaration des nœuds eulériens et lagrangiens ;
- distribution de la masse nodale de la structure sur les nœuds des éléments eulériens;

- distribution de la force nodale de la structure sur les nœuds des éléments eulériens ;

- calcul des nouvelles accélérations des nœuds eulériens ;

- calcul des nouvelles accélérations des nœuds de la structure en fonction des nouvelles accélérations des nœuds eulériens ;

- distribution de la quantité de mouvement nodale de la structure sur les nœuds des éléments fluides ;

- calcul des nouvelles vitesses nodales du fluide;

- calcul des nouvelles vitesses nodales de la structure en fonction des nouvelles vitesses nodales du fluide;

- retour des nouds eulériens à leur position initiale et début d'une étape d'advection;

- traitement des éléments lagrangiens (déformation, contraintes) et déformation du maillage;

- calcul des nouvelles positions du maillage lagrangien;

- reprise d'un nouveau cycle de calcul.

\subsection{Lois de comportement pour le jet et la cible}

Le code de calcul LS-DYNA dispose de modèles qui simulent le comportement des matériaux «fluides » et qui sont tous des modèles isotropiques. Nous trouvons notamment des matériaux à comportement de type « Null material » que nous avons utilisé pour simuler le comportement de la partie liquide (eau + air). Ce modèle est caractérisé par un tenseur de contraintes qui est la somme des contraintes de pression (tenseur sphérique) et de viscosité (tenseur déviateur). 
Tableau 2. Valeurs des propriétés des fluides utilisés dans le maillage eulérien.

\begin{tabular}{cccc}
\hline Fluide & $\begin{array}{c}\text { Masse volumique } \\
(\rho)\left(\mathrm{kg} / \mathrm{m}^{3}\right)\end{array}$ & $\begin{array}{c}\text { Pression de coupure } \\
(\text { cut-off pressure }) \\
(P c)(\mathrm{Pa})\end{array}$ & $\begin{array}{c}\text { Viscosité dynamique } \\
\left(\text { à } 20{ }^{\circ} \mathrm{C} \text { et pression atmosphérique }\right) \\
(\mu)(\text { Pa.s })\end{array}$ \\
\hline Eau & 1000 & $-10^{5}$ & $10^{-3}$ \\
\hline Air & 1,29 & -10 & $1,67 \times 10^{-5}$ \\
\hline
\end{tabular}

Tableau 3. Coefficients $K_{(i=1 \ldots 6)}$ relatifs à l'air et à l'eau.

\begin{tabular}{llllllll}
\hline & $K_{0}$ & $K_{1}$ & $K_{2}$ & $K_{3}$ & $K_{4}$ & $K_{5}$ & $K_{6}$ \\
\hline Eau & 0 & 2,2 & 9,54 & 14,57 & 0,28 & 0,28 & 0 \\
\hline Air & 0 & 0 & 0 & 0 & 0,401 & 0,401 & 0 \\
\hline
\end{tabular}

Tableau 4. Valeurs des propriétés physiques de la cible.

\begin{tabular}{cllll}
\hline Matériaux & $\rho\left(\mathrm{kg} / \mathrm{m}^{3}\right)$ & $E(\mathrm{GPa})$ & $\nu$ & $R_{\mathrm{y}}(\mathrm{MPa})$ \\
\hline A2024 & 2700 & 73 & 0,33 & 345 \\
\hline Polyuréthane & 1200 & 0,9 & 0,25 & 40 \\
\hline
\end{tabular}

La mise à jour des contraintes de pression est assurée par la relation donnant l'équation d'état du fluide, alors que la réactualisation du terme déviateur est donnée par le calcul du taux de déformation dans le fluide. Nous précisons que la pression statique dans le fluide admet une borne inférieure appelée «pression de coupure » qui est négative (Tab. 2).

L'équation d'état associée à la loi hydrodynamique traduisant le comportement fluidique, définit une relation entre la pression, la masse volumique et l'énergie interne spécifique (Éq. (3)). Dans notre cas, nous avons utilisé l'équation d'état dite « équation d'état polynomiale linéaire $»$, et ceci pour chaque fluide (eau, air). Cette équation se présente sous la forme suivante :

$$
\begin{aligned}
p=K_{0}+K_{1} \alpha+K_{2} \alpha^{2}+ & K_{3} \alpha^{3} \\
& +\left(K_{4}+K_{5} \alpha+K_{6} \alpha^{2}\right) e
\end{aligned}
$$

avec

$p$ : pression,

$e$ : énergie interne par volume initial (dite encore spécifique),

$K_{0}, K_{1}, K_{2}, K_{3}, K_{4}, K_{5}$ et $K_{6}$ : constantes dépendant du matériau (Tab. 3),

$\alpha$ : fonction du volume relatif $V, \alpha=\left(\frac{1}{V}-1\right)$.

En ce qui concerne la cible, elle est régie par un modèle élasto-plastique caractérisé par une densité de déformation qui se décompose en une partie élastique et une partie plastique. Pour le comportement plastique, nous nous limitons au cas de la plasticité associée avec un critère de von Mises et un écrouissage isotrope [16]. Les caractéristiques de chacun des matériaux de la cible, ( $\rho$ : masse volumique, $E$ : module d'Young, $\nu$ : coefficient de poisson, $R_{y}$ : contrainte limite élastique) sont données par le tableau 4.

\subsection{Conditions aux limites et chargement}

Deux types de conditions aux limites sont pris en compte. Le premier type concerne le maillage eulérien. Dans ce cas, une condition de frontières libres est définie. Une distribution dynamique de vitesse $(500 \mathrm{~m} / \mathrm{s})$ est aussi introduite afin de simuler le jet (Fig. 5). Le deuxième type concerne des conditions aux limites lagrangiennes. En effet, des conditions frontières absorbantes sont introduites afin d'éliminer la réflexion des ondes qui peuvent perturber l'interaction jet-cible. Le modèle est ainsi considéré comme infini. Nous avons supposé que l'interface revêtement-substrat soit parfaite. La base de la cible est supposée appuyée sur un plan (les mouvements des nœuds selon l'axe $Z$ sont prohibés). Il est à noter que nous ne présentons pas dans cette étude le cas de la cible siège de phénomène de flexion où des mouvements de vibration peuvent se manifester. Ce dernier cas correspond à une interaction assez complexe et qui mérite une étude à part. Dans notre étude, la cible (revêtement+substrat) est animée par une vitesse linéaire égale à $5,7 \mathrm{~m} / \mathrm{s}$ une fois exposée au jet. Cela traduira implicitement la rotation d'une tête monojet avec une cible à décaper.

\section{Résultats et discussion}

Après avoir décrit les outils numériques qui permettent de modéliser l'interaction jet-cible nous présentons les résultats numériques relatifs à cette interaction. Le cas d'un jet de vitesse $500 \mathrm{~m} / \mathrm{s}$ qui décape le revêtement $(100 \mu \mathrm{m})$ d'une plaque homogène infinie (A2024, épaisseur $1 \mathrm{~mm}$ ) est étudié. La vitesse de la cible est égale à $5,7 \mathrm{~m} / \mathrm{s}$.

\subsection{Comportement du jet durant l'opération de décapage}

Cette partie concerne l'évolution du champ de vitesse du jet précédent, durant et à la fin de son interaction avec le couple revêtement - substrat.

Nous notons que le temps nécessaire pour que le jet puisse atteindre la cible est de $1 \mu \mathrm{s}$. Pendant cette période le jet garde une forme cylindrique. Ensuite, il heurte la cible et provoque un état de choc caractérisé par des instabilités qui durent $0,02 \mu \mathrm{s}$ (après l'instant d'impact) [12]. Le jet subit alors des réflexions (Figs. 6 et 7) qui à leur tour animent, en vitesse, la zone eulérienne du modèle. Nous remarquons aussi la présence de deux zones de recirculation de vitesse très prononcées surtout à droite du jet. 


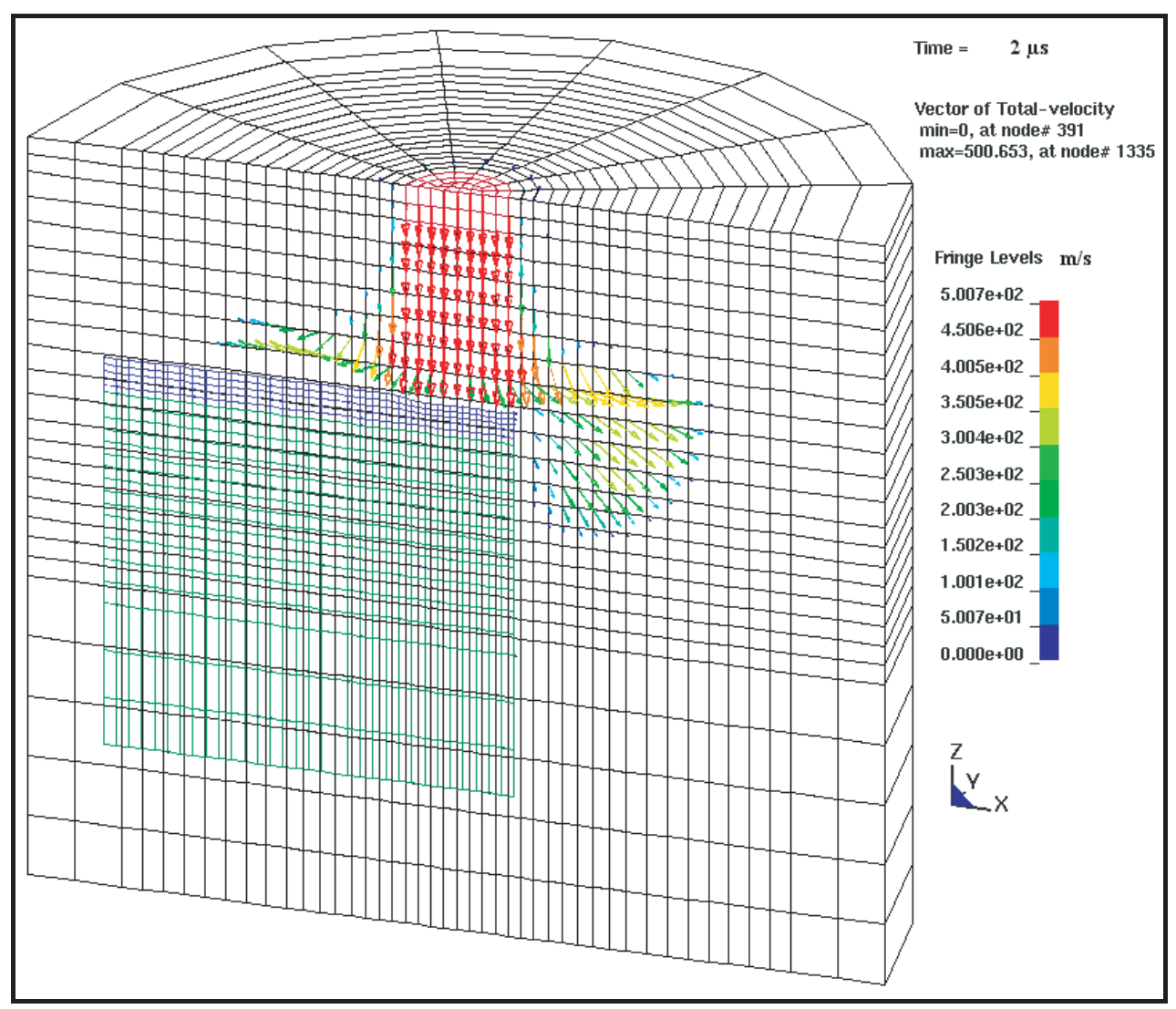

Fig. 6. Début de l'opération de décapage (la cible se déplace de gauche à droite).

Les figures 6-9 montrent, respectivement le début et la fin de l'opération de décapage. Pendant ces deux étapes, nous pouvons assister à des altérations de bord sur les pièces à traiter. En effet, le jet présente une partie sur la pièce et une autre à l'extérieur.

Dans le cas d'un jet immobile par rapport à la pièce, il a été démontré précédemment [11, 12] qu'il existe une zone de stagnation fluide sur l'axe située dans l'écoulement axial du jet, qui semble être à l'origine de la formation d'un bossage au centre de l'impact générée. La région liquide entourant la zone centrale de l'impact est animée par un écoulement radial assez destructif qui est à l'origine de la génération des contraintes de cisaillement au sein de la matière à décaper. Ainsi, la structure hydrodynamique du jet est d'une importance primordiale quant à la morphologie des impacts créés.

\subsection{Comportement de la cible durant l'opération de décapage}

Cette partie est consacrée à l'étude de l'évolution du champ de contrainte généré pendant l'interaction jetcible. Nous présentons les distributions des contraintes dans la cible et ceci selon des plans de sections transversales (Fig. 10).

La figure 11 illustre la distribution des iso-valeurs des contraintes $Y Z$ au sens du critère de von Mises ainsi que le champ de vitesse fluide selon la section transversale (A1-A2). Le temps d'exposition de la cible au jet est égal à $2 \mu \mathrm{s}$. Cette étape d'interaction est caractérisée par la présence d'un état de cisaillement au sein du revêtement dont la valeur maximale est égale à 22,61 MPa. Pendant ce temps, aucun phénomène d'érosion n'est observé. La morphologie de l'empreinte sous le chargement du jet, présente un bossage au centre (Fig. 11). Ce dernier est entouré par une zone annulaire. Cette morphologie n'est pas stationnaire, au cours du décapage, pour des temps d'exposition plus élevés (Fig. 12).

La figure 12 correspond à une visualisation des contraintes de von Mises au sein du revêtement pour des temps d'exposition égaux à $118 \mu$ s et $174 \mu$ s. Dans ce cas de figure, nous notons un maximum de contrainte localisé sur la périphérie de la zone décapée. Cela prouve l'efficacité de l'aplatissement du jet qui génère des contraintes de cisaillement au sein du revêtement et par conséquent son érosion. Ce phénomène semble être prépondérant lors d'une opération de décapage. Par ailleurs, nous notons la présence de contraintes localisées le long de la direction $(X X)$ : zone soumise au passage de la partie centrale du jet. Comme il sera précisé plus tard, cette zone peut montrer des fissurations discontinues sur la ligne médiane du décapage.

La figure 13 montre la distribution des contraintes de von Mises dans le substrat (A2024) (dans cette figure le revêtement n'est pas illustré). Nous notons que le niveau 


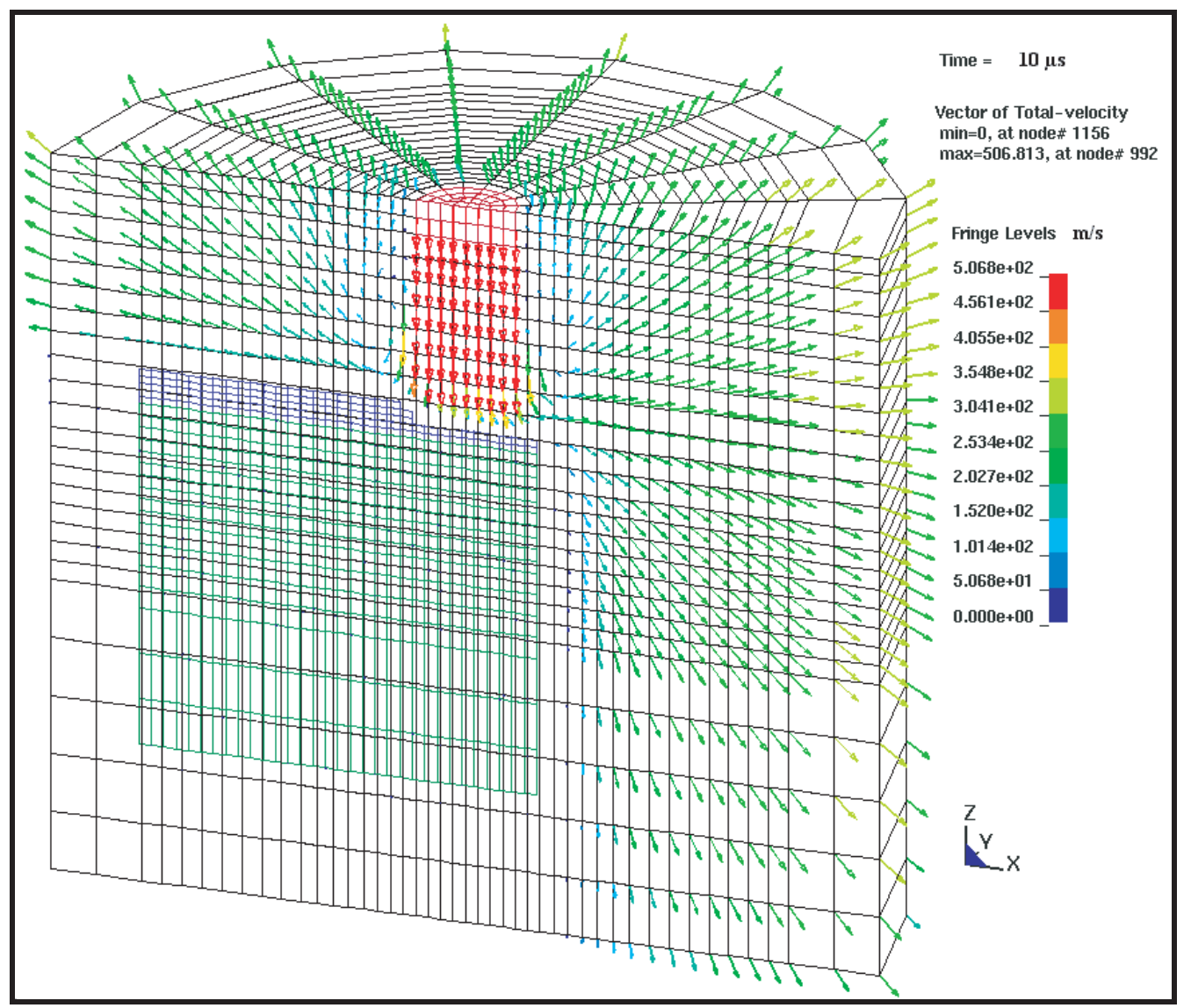

Fig. 7. Etablissement de l'opération de décapage. Observation du phénomène d'érosion.

des contraintes résiduelles introduit par le passage du jet atteint $166 \mathrm{MPa}$. Les iso-valeurs de ces contraintes sont surtout localisées le long de l'axe $(X X)$.

La figure 14 correspond à la représentation des isovaleurs des contraintes de cisaillement $(Z X)$ dans le substrat et pour un temps d'exposition de $118 \mu \mathrm{s}$. Les contraintes sont plus importantes dans la zone de gauche que celle de droite. Cela est du probablement au fait que, lors de son écoulement sur la cible suivant la direction $(-X X)$, le jet génère des contraintes opposées à celles générées par le mouvement de la cible $(+X X)$. D'où une augmentation de l'état de contrainte de cisaillement du coté gauche du jet. Par contre, dans la zone de droite le mouvement de la cible et l'écoulement du jet ont le même sens. En examinant la figure 15 nous constatons que les valeurs des contraintes $Y Z$ sont positives. Ceci est expliqué par l'effet de raclage introduit par l'écoulement latéral du jet quand il est en contact avec la cible.

La figure 16 montre diverses étapes de l'érosion du revêtement jusqu'à un temps d'exposition de $174 \mu \mathrm{s}$. A $2 \mu$ s nous notons que le revêtement subit une déformation plastique. Après ce temps, l'érosion est générée. Dans ce cas d'impact normal, le substrat n'est pas encore atteint et le jet décape une couche d'épaisseur approximative de $60 \mu \mathrm{m}$. Suivant les figures 14 et 15, l'action la plus importante qui favorise cette érosion est celle produite par l'aplatissement du jet sur la cible. Afin de modifier les conditions hydrodynamiques de l'écoulement du jet sur la cible, nous avons incliné le jet d'un angle de 15 degrés (Fig. 17). Dans ce cas d'écoulement, nous remarquons que l'érosion du revêtement est devenue plus importante par rapport au cas d'un jet décapant normal (Fig. 19). La distribution hydrodynamique aux alentours de l'interaction jet incliné- cible est caractérisée par la formation d'une zone de stagnation prononcée derrière le jet (Fig. 18). Nous notons que d'après les travaux de Rochester [20], le maximum de l'enlèvement de matière provoqué par un jet heurtant une cible correspond à un angle d'incidence du jet variant entre 15 et 20 degrés.

\section{Résultats expérimentaux}

Un des paramètres importants pour le décapage par jet d'eau HP est le temps d'exposition de la structure à décaper au jet. Le système de mise en route du jet, dont nous disposons, ne permet pas d'avoir un pilotage du temps de tir précis (quelques dixièmes de seconde au mieux). Un tel temps est élevé pour étudier finement un impact généré par un jet en mouvement ou immobile par rapport à une cible revêtue.

Afin de mieux contrôler ce temps d'exposition de la pièce au jet nous avons conçu un montage qui consiste à utiliser deux disques $\left(D_{1}, D_{2}\right)$ en partie superposés et 


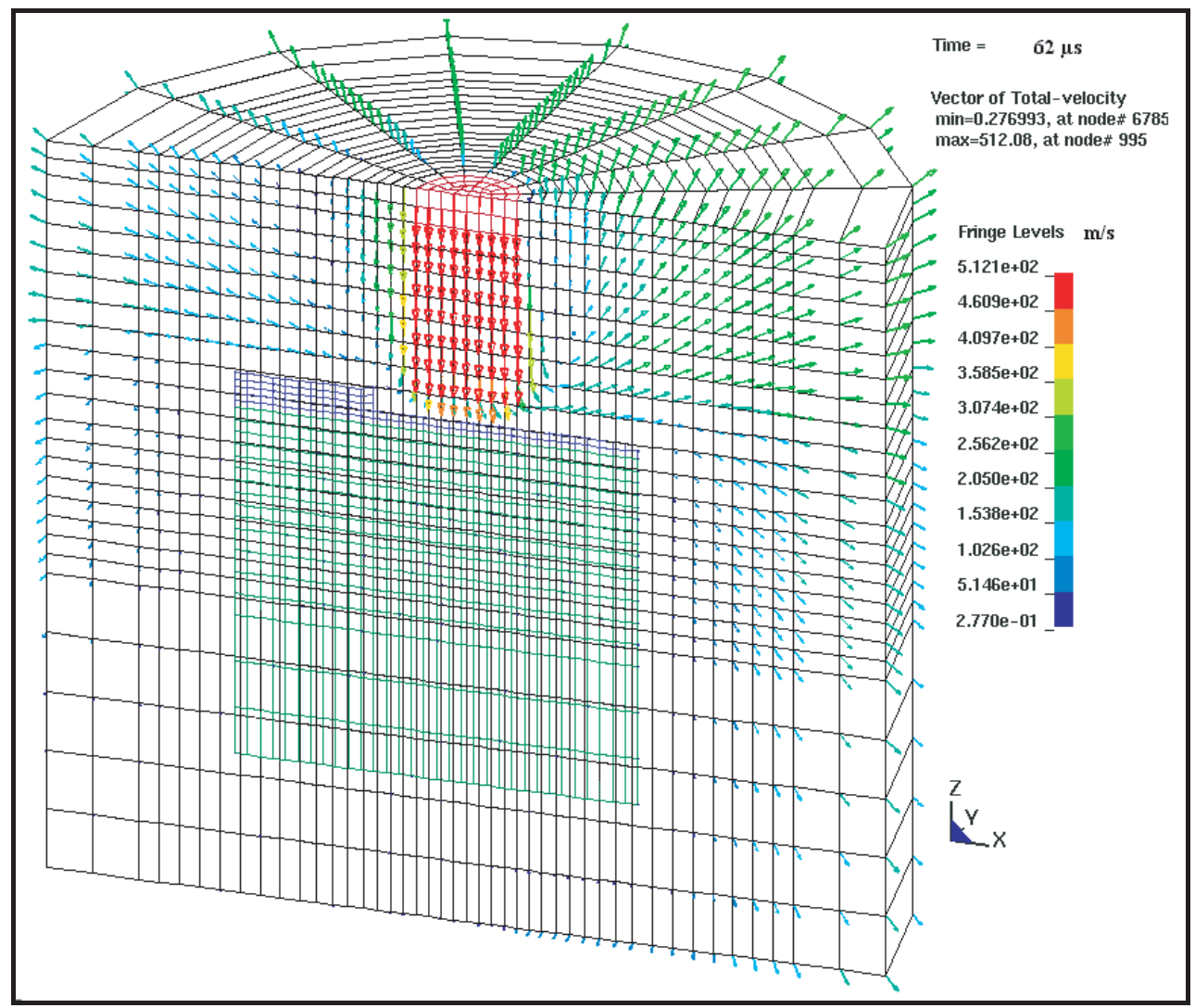

Fig. 8. Présence de deux zones de recirculation de vitesse très prononcée à droite du jet.

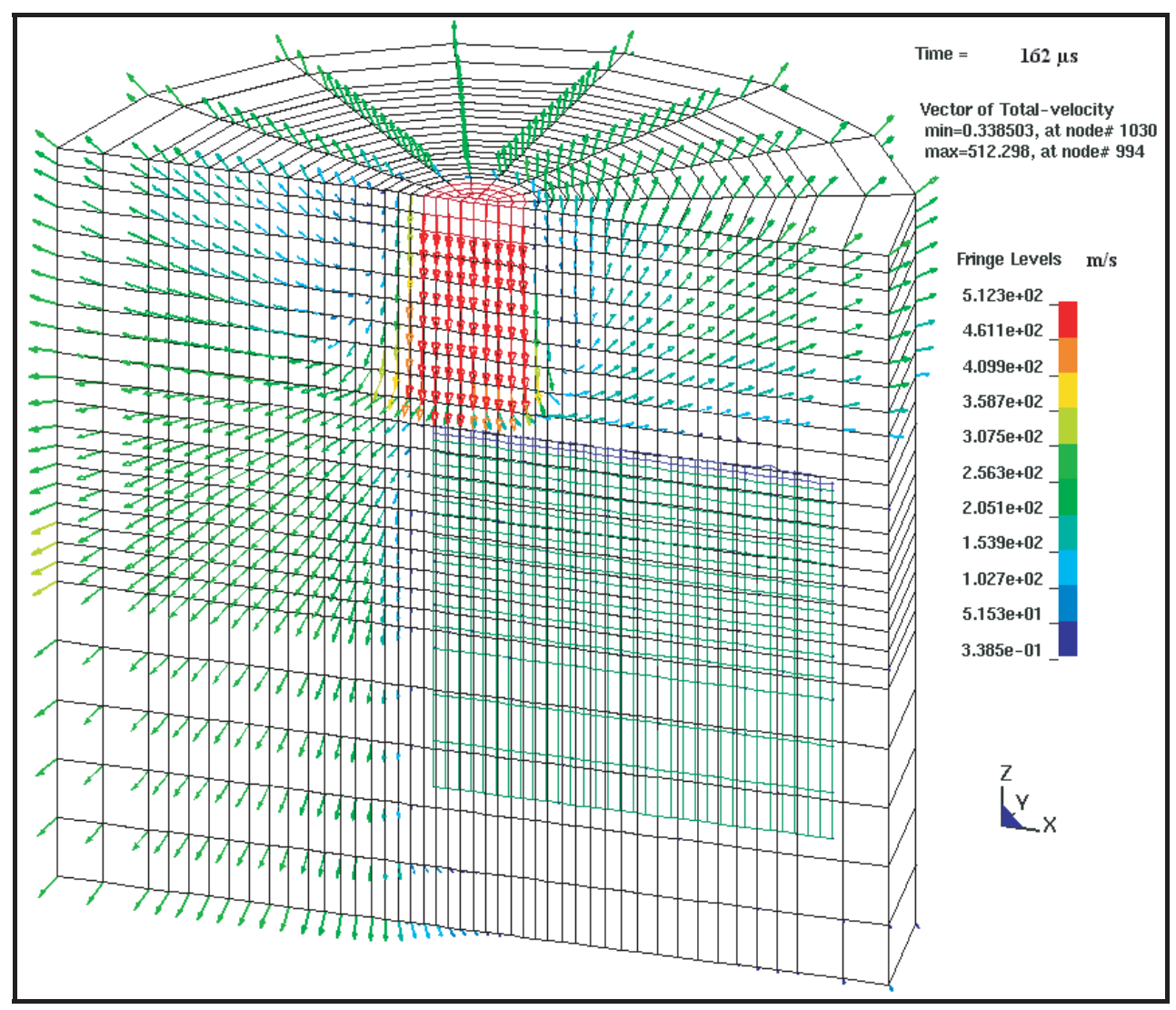

Fig. 9. Début de la fin du décapage. 


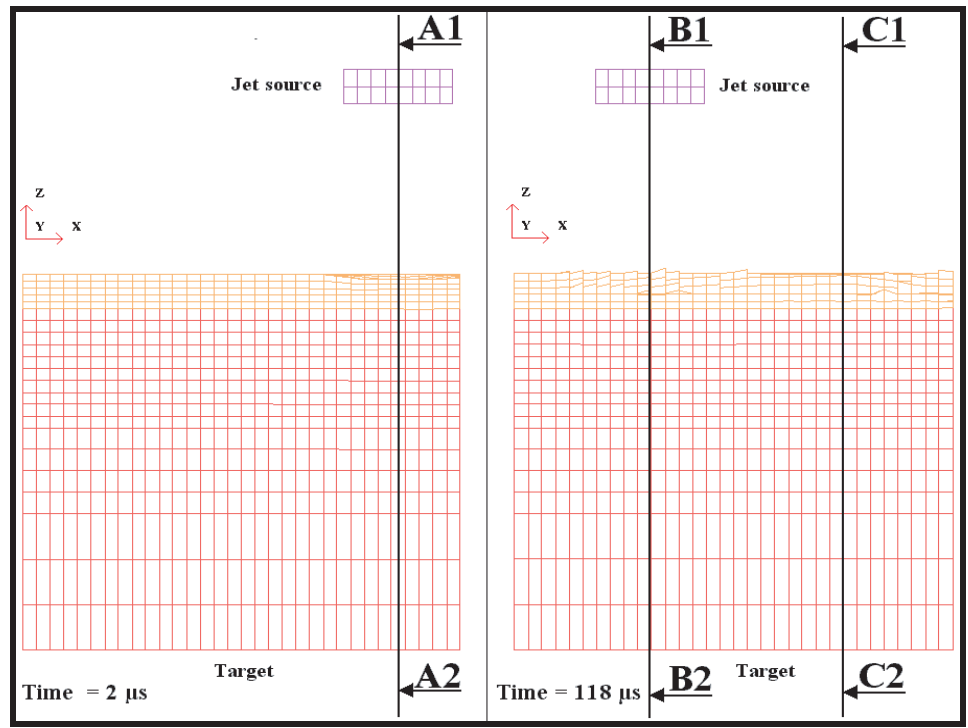

Fig. 10. Positionnement des plans de sections transversales.

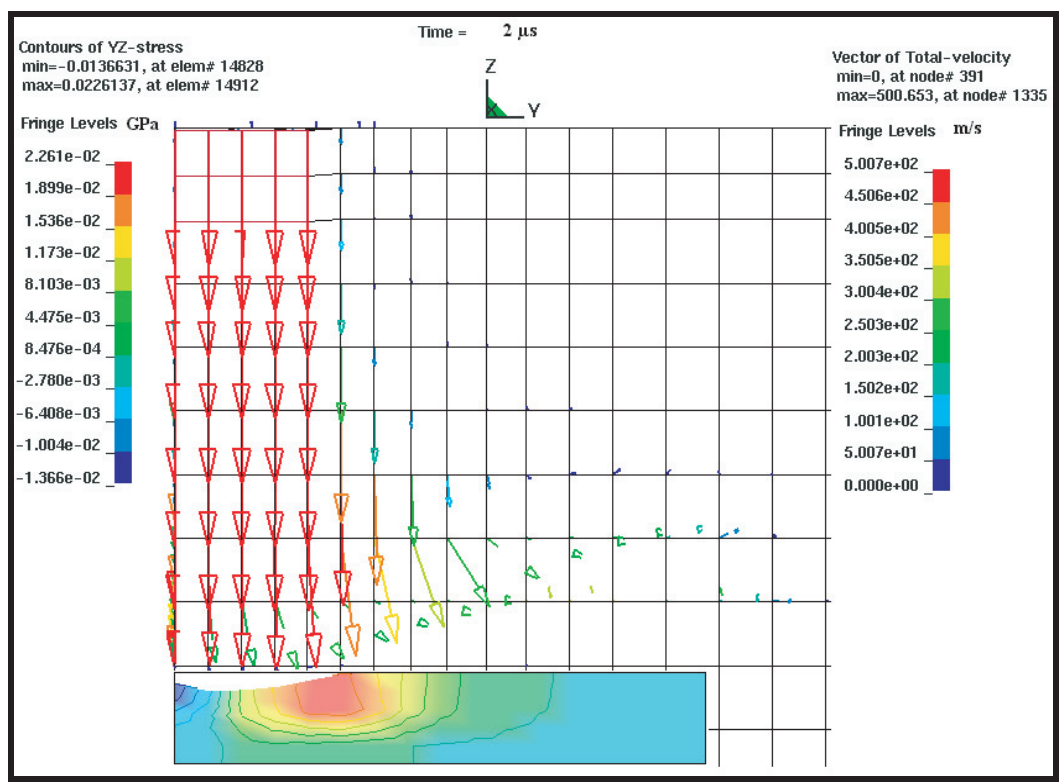

Fig. 11. Distribution des contraintes de von Mises $(Y Z)$ et du champ de vitesse fluidique visualisé dans la section transversale $(\mathrm{A} 1-\mathrm{A} 2)($ temps $=2 \mu \mathrm{s})$.

entraînés en rotation par un moteur à courant continu. Chacun est doté d'une fente de dimensions et de forme particulière (Fig. 20). La maîtrise du temps d'exposition est obtenue par la superposition de l'ouverture de chacune des deux fentes et varie grâce à la vitesse de rotation du moteur [12].

Avec ce montage nous avons pu étudier précédemment [8-12] l'interaction d'un jet immobile avec des cibles revêtues (A2024 revêtue par une peinture polyuréthane et E24 revêtue par une peinture époxydique).

La génération du jet HP a été réalisée par l'intermédiaire d'une pompe qui fournit une pression jusqu'à $380 \mathrm{MPa}$. Le jet sort dans le milieu ambiant à travers une buse de diamètre $0,3 \mathrm{~mm}$. La distance de tir est fixée à $d_{\mathrm{tir}}=30 \mathrm{~mm}$. Avec ces conditions, et grâce au montage précédent, on a pu réaliser des traînées de décapage résultat de l'impact du jet avec l'échantillon. Ces empreintes permettent de simuler une trace produite lors d'un décapage réalisé par une tête rotative à une seule buse (Fig. 2); l'échantillon (plaque A2024 revêtue par une couche en polyuréthane $(100 \mu \mathrm{m}))$ étant fixé en bas du disque $\left(\mathrm{D}_{1}\right)$ (Fig. 20) animé par une vitesse linéaire égale à $5,7 \mathrm{~m} / \mathrm{s}$. Ces conditions expérimentales correspondent aux paramètres utilisés au cours de la modélisation déjà présentée ci-dessus.

Un exemple de résultat correspondant aux conditions précédentes est donné par la figure 21a. La morphologie 


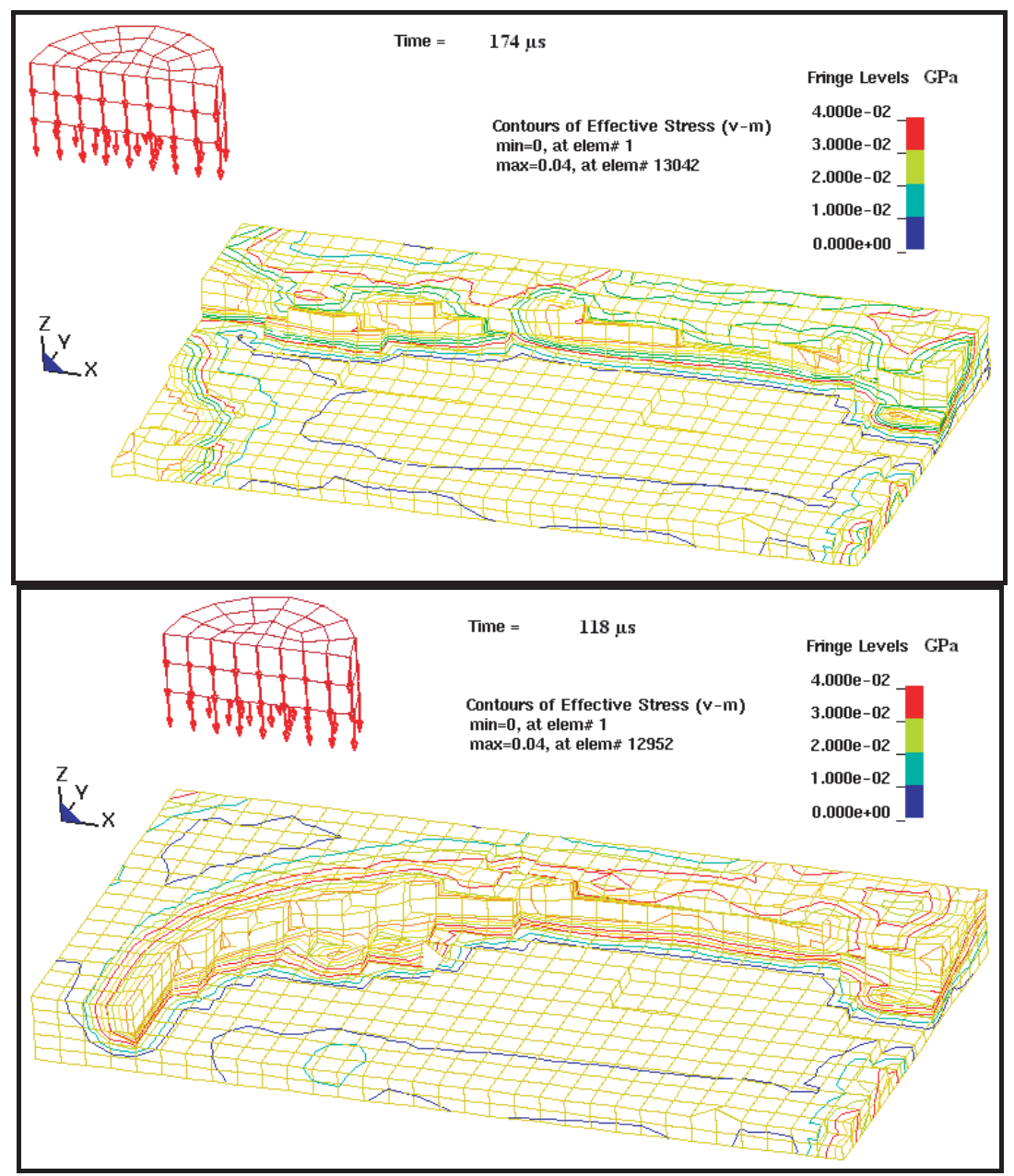

Fig. 12. Distributions des contraintes de von Mises au sein du revêtement traité.

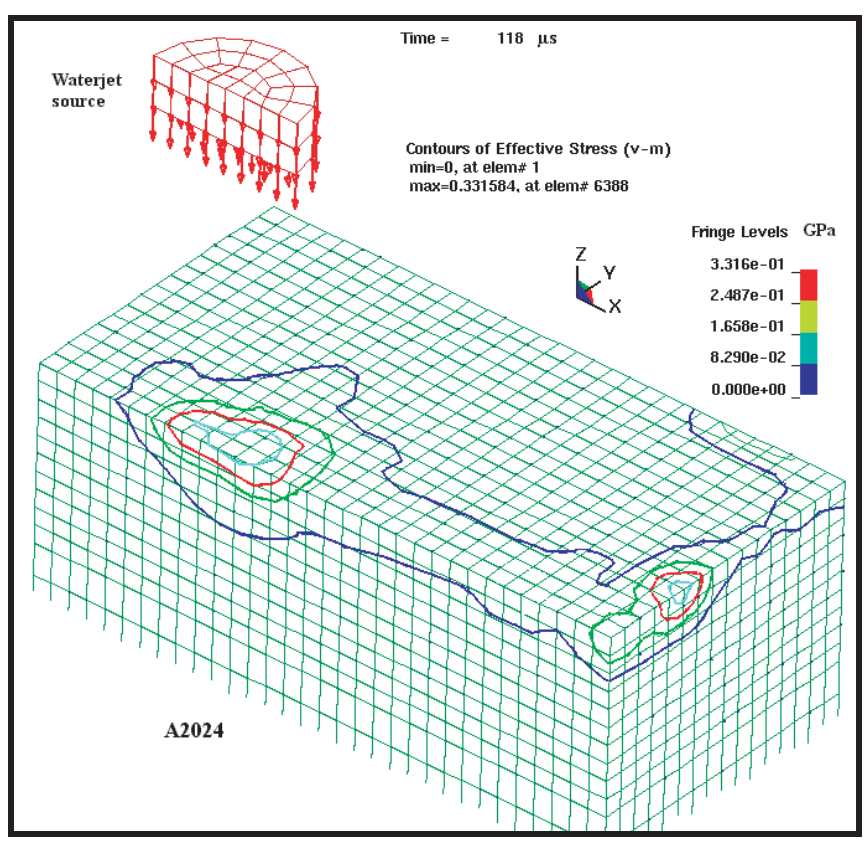

Fig. 13. Distribution des contraintes de von Mises au sein du substrat.

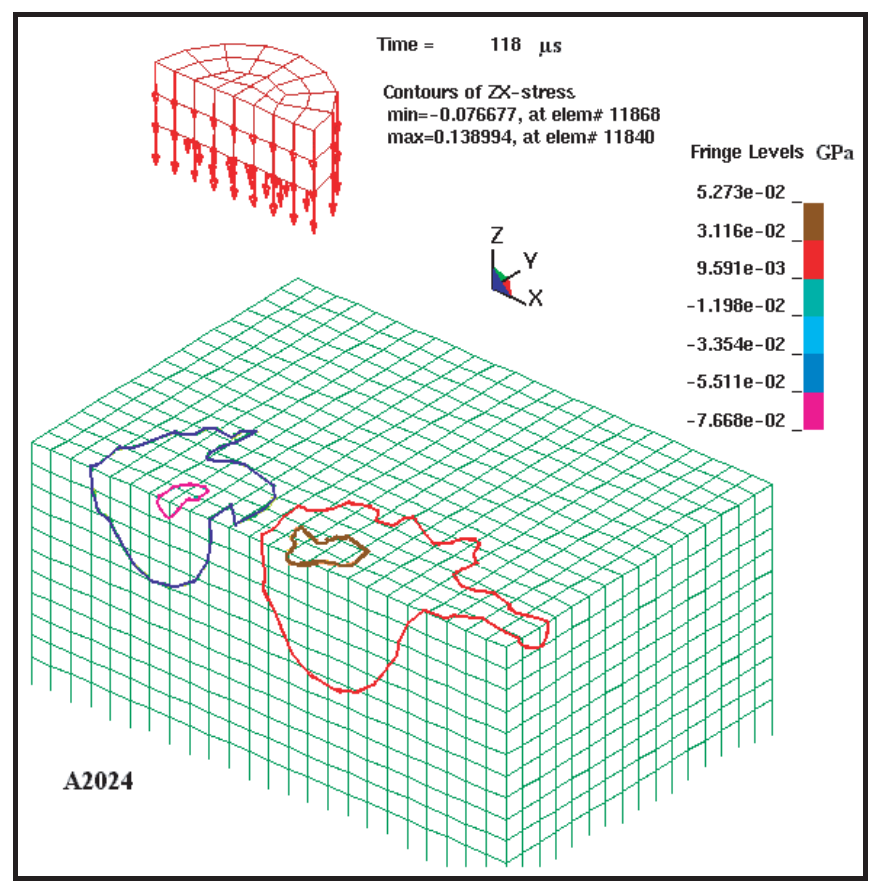

Fig. 14. Distribution des contraintes de von Mises $(Z X)$ au sein du substrat. 


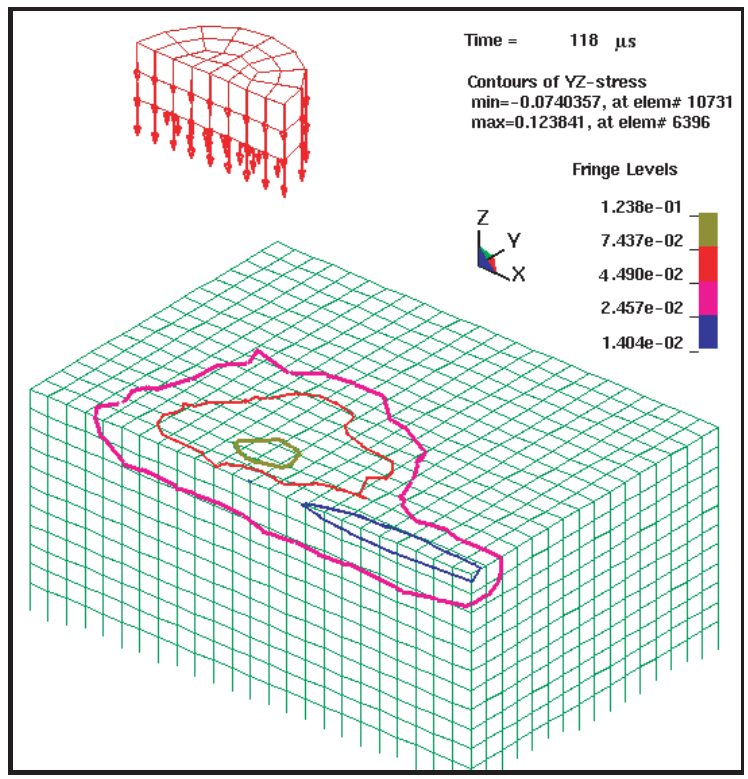

Fig. 15. Distribution des contraintes de von Mises $(Y Z)$ au sein du substrat.

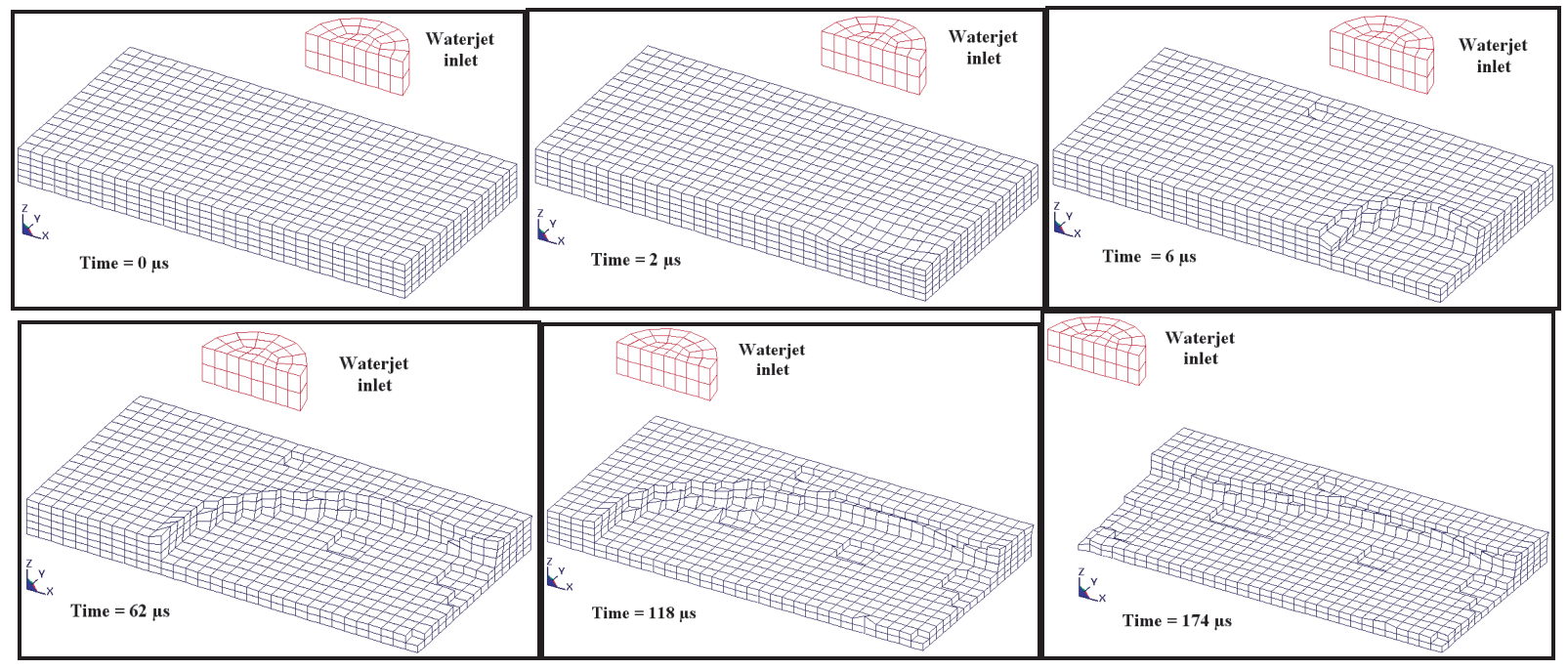

Fig. 16. Erosion du revêtement en fonction du temps d'exposition.

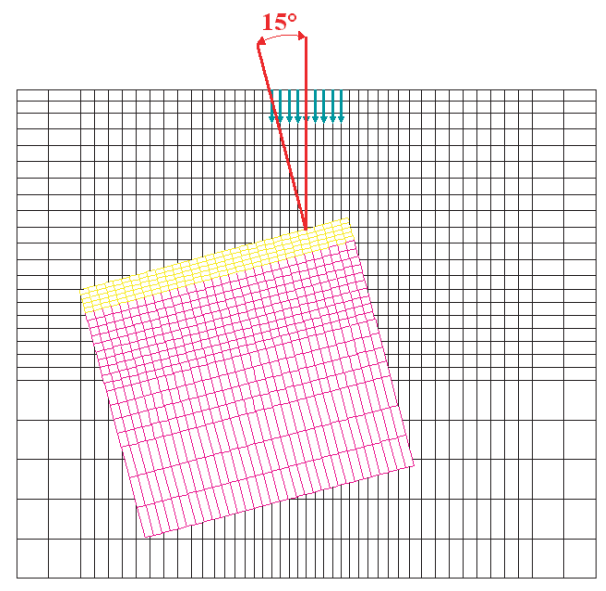

Fig. 17. Jet avec angle d'incidence. du décapage généré, selon la largeur de l'empreinte, est montrée par la figure 21b. L'élargissement du décapage est gouverné par l'efficacité du jet, lors de son aplatissement, à éroder la matière. Nous remarquons, en outre, que les valeurs en largeur et en profondeur du décapage sont proches de celles trouvées numériquement (Fig. 21c).

Afin de mieux comprendre le mode d'enlèvement du revêtement, une observation microscopique de l'empreinte résultant de l'action du jet sur la cible est effectuée (Fig. 22). En examinant la figure 22, en plus du phénomène d'érosion il y a une présence de quelques fissurations discontinues sur la ligne médiane de la zone décapée. Ce comportement est provoqué par l'étirement opposé subi par le revêtement sous l'action de l'aplatissement du jet sur la cible. Les résultats numériques montrés par la figure 15, confirment ce comportement. En effet, l'aplatissement du jet est un facteur très important quant 


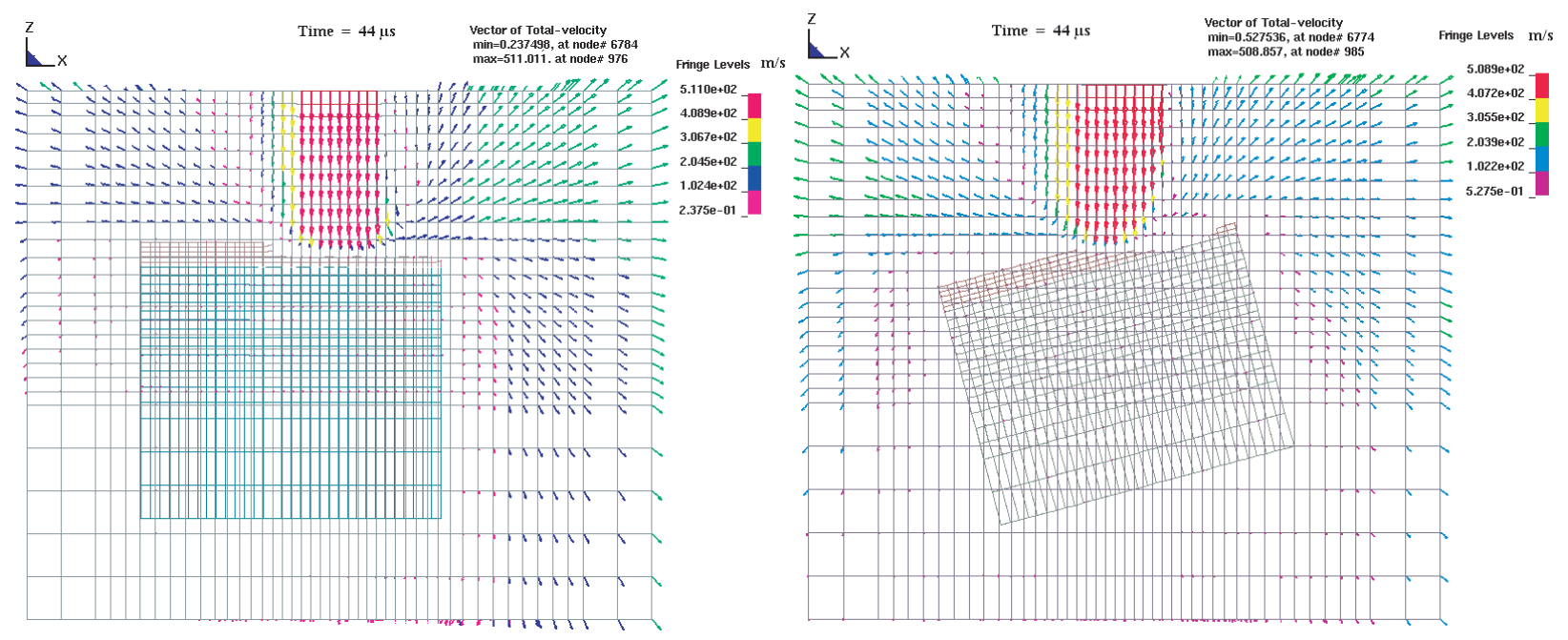

Fig. 18. Influence de l'angle d'incidence sur l'hydrodynamique du jet.
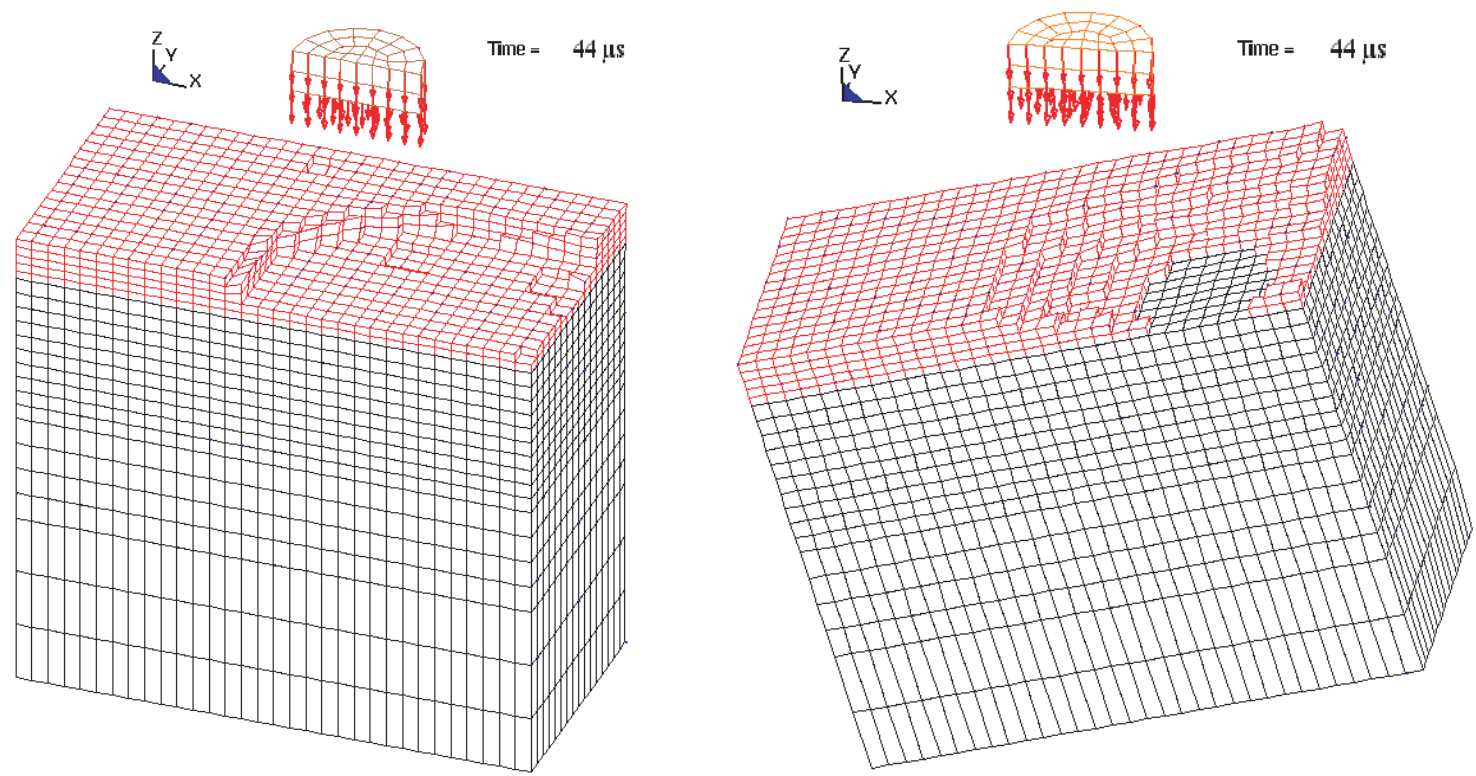

Fig. 19. Influence de l'angle d'incidence du jet sur l'érosion de la cible.

au processus du décapage par jet d'eau. En conséquence, il apparaît clairement, pour ce type de revêtement, qu'en plus de l'effet prépondérant et érosif du jet sur la cible, le processus d'enlèvement de ce revêtement est accéléré par l'apparition de fissurations. En effet, ces dernières peuvent être des sites privilégiés traversés par des micro-jets accélérant le décollement du revêtement et par conséquent améliorant l'opération de décapage.

\section{Conclusion et perspectives}

Bien que le procédé de décapage par jet d'eau haute pression soit de plus en plus répandu dans le monde industriel, il souffre encore d'un manque d'outils fiables. Ce constat nous a amené à traiter ce procédé à son niveau basique qui est l'interaction du jet avec le composé revêtement-substrat. Nous souhaitons, bien évidemment, apporter des explications cohérentes à la phénoménologie de cette interaction qui pourront ultérieurement servir au développement de ces outils. L'objectif du travail présenté à travers cet article est de comprendre le mode d'enlèvement d'un revêtement lors d'une opération de décapage par jet d'eau HP. Pour cela, nous avons mis en place un modèle numérique traitant l'interaction jet-cible par l'intermédiaire du code éléments finis LSDYNA. Plusieurs restrictions au niveau de la simulation ont été optées (interface parfaite, enlèvement de matière basé seulement sur un critère d'allongement à la rupture des mailles, manque de modèle de turbulence dans la partie fluidique du modèle). Cependant, nous avons obtenu des résultats qui confirment nos constatations 

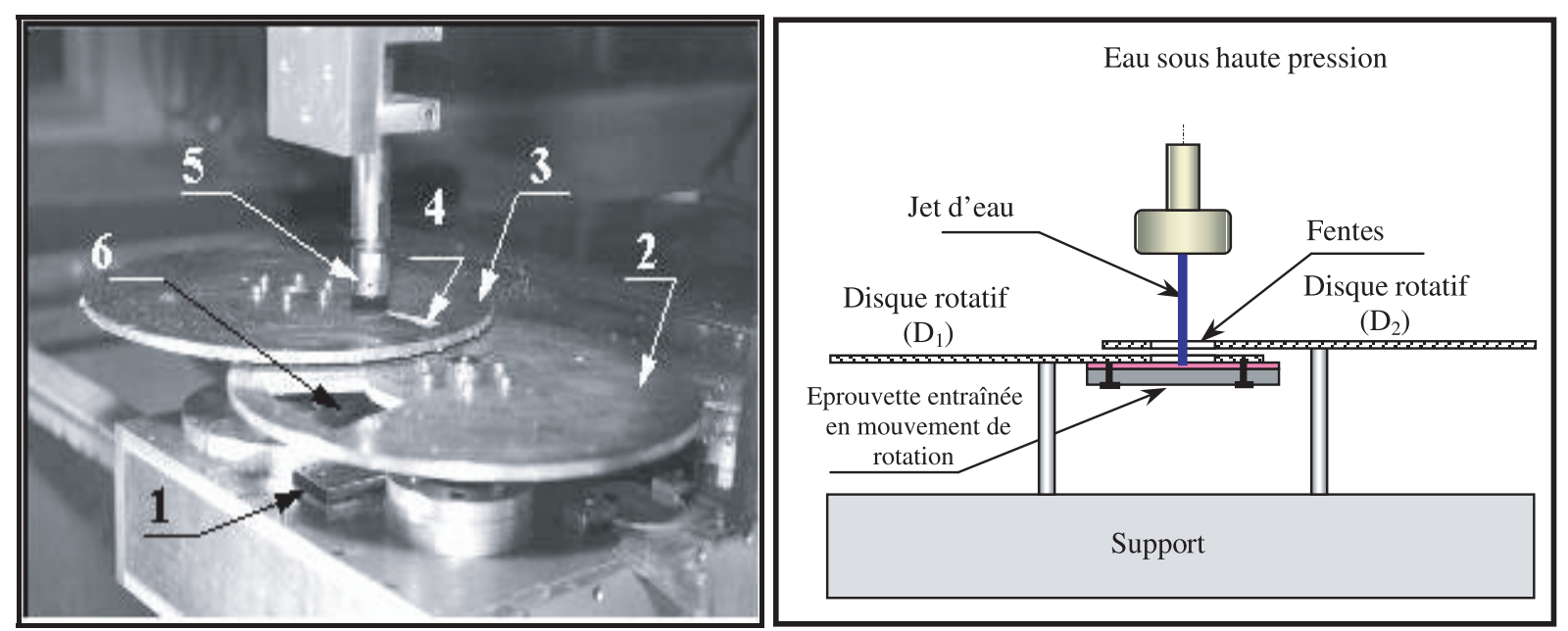

Fig. 20. Montage expérimental de mesure du temps d'exposition d'une cible à un jet d'eau HP (éprouvette (1), disques rotatifs $(2,3)$, buse $(5)$, fentes $(4,6))$.

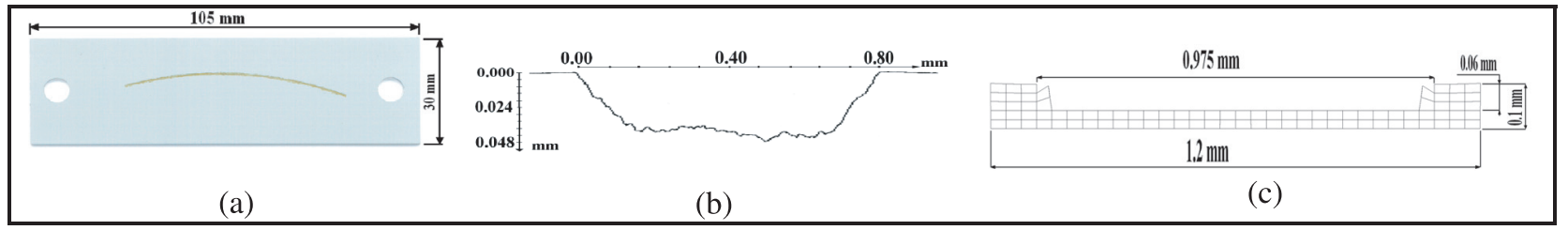

Fig. 21. Morphologies des empreintes de décapage : résultats expérimentaux et numériques.
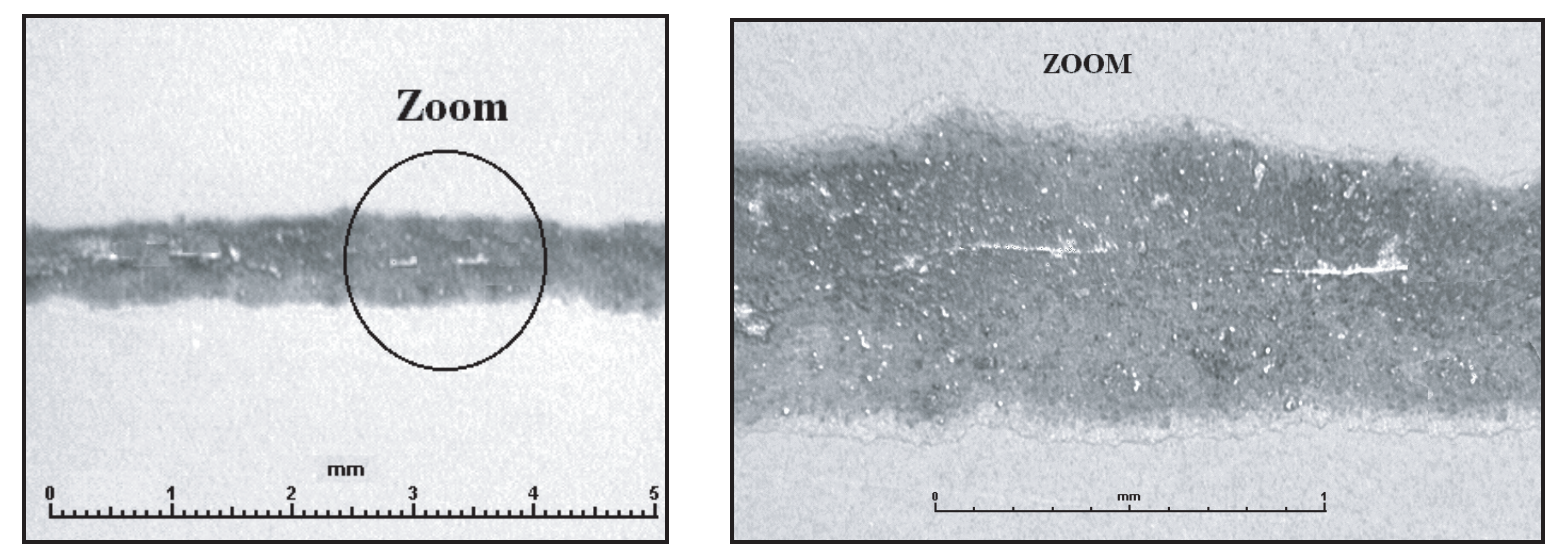

Fig. 22. Observations microscopiques de l'empreinte de décapage sur le polyuréthane (présence de fissurations sur la ligne médiane).

expérimentales. En effet, la simulation montre l'importance de l'écoulement latéral du jet, qui crée un effet de tension radiale dans le revêtement, ce qui provoque son endommagement. L'érosion numérique introduite dans le modèle semble être réaliste pour simuler le phénomène prépondérant lors d'une opération de décapage. Nous remarquons, en outre que l'incidence du jet joue un rôle important sur son efficacité. Il semble qu'un angle d'incidence de 15 degrés provoque un enlèvement de revêtement plus important qu'une incidence normale. Un montage expérimental a été conçu afin de bien contrôler l'interaction jet-cible. Des techniques d'investigation nous ont permis d'effectuer des observations fines sur des pièces décapées. Il s'est avéré qu'en plus du phénomène d'érosion il peut y avoir au cours de l'interaction jet-cible une génération de micro-fissurations. Ces dernières peuvent être le siège d'une pénétration hydraulique (micro-jets) dans le revêtement favorisant ainsi son décollement.

La suite de notre travail visera à étudier l'interaction de plusieurs jets en mouvement par rapport à la cible. Une attention particulière cela prise pour étudier les phénomènes qui accompagnent les interactions successives des jets. Nous adopterons les deux voies expérimentale et numérique et ceci afin de mettre en place un modèle prédictif de l'enlèvement de matière dans ce cas de procédé de décapage. 


\section{Références}

[1] H. Louis, W. Schikorr, Fundamental aspects in cleaning with high-speed waterjets, Proc 6th Int Conf Jet Cutting Techn. Guildford (1982) 217-228

[2] A.F. Conn, Waterjet cleaning for in-factory application, Proc. 11th Int. Conf. Jet Cutting Techn. Dordrecht (1992) 443-449

[3] S.S. Wu, T.J. Kim, An application study of plain waterjet process for coating removal, Proc 8th Int. Conf. Jet Cutting Techn. Houston (1995) 779-792

[4] P.L. Kaye, C.S.J. Pickles, Field J.E., Julian K.S., Investigation of erosion process as cleaning mechanism in the removal of thin deposited soils, Wear 186-187 (1995) 413-420

[5] M.C. Leu, P. Meng, E.S. Geskin, L. Tismeneskiy, Mathematical modeling and experimental verification of stationary waterjets cleaning process, Trans. ASME J. Manuf. Sc. Engin., 120 (1998) 571-579

[6] P. Meng, E.S. Geskin, M.C. Leu, F. Li, L. Tismeneskiy, An analytical and experimental study of cleaning with moving waterjets, Trans. ASME J. Manuf. Sc. Engin., 120 (1998) 580-589

[7] G.S. Springer, Erosion by Liquid Impact. Scripta Publishing Co., Washington, DC, (1976)

[8] T. Mabrouki, A. Cornier, K. Raissi, Experimental investigation and numerical modeling of HP pure waterjet impingement in the aeronautical decoating process, 13th Int. Conf. Surf. Treat. Aeron. Aeros. Ind. Surfair 2000, (Cannes 14-16 June 2000)

[9] T. Mabrouki, A. Cornier, K. Raissi, The study of HP pure waterjet impact as the primary mechanism of paint decoating process, 14th Int. Conf. Jetting Techn. (Brugge Belgium 21-23 September 1998) pp. 563-577
[10] T. Mabrouki, K. Raissi, A. Cornier, Numerical simulation of high velocity pure waterjet impingement on coated material. 15th Int. Conf. Jetting Techn. (Ronneby Sweeden, 6-8 September 2000) pp. 199-217

[11] T. Mabrouki, exploration expérimentale et modélisation numérique des impacts fluidiques: Contribution à l'étude du décapage par Jet d'eau pure HP, Thèse de Doctorat ENSAM-Paris-France (6 juin 2000)

[12] T. Mabrouki, K. Raissi, A. Cornier, A numerical simulation and experimental study of the interaction between a pure high-velocity waterjet and targets : contribution to investigate the decoating process. Wear 239 (2000) 260-273

[13] K. Yanaida, Flow characteristics of water jets. Proc. 2nd Int. Symp. Jet Cutting Tech., BHRA Fluid Engng., Cranfield (1974) A2/19-A2/32

[14] K.F. Neusen, T.G. Gores, R.S. Amano, Axial variation of particle and drop velocities downstream from an abrasive water jet mixing tube, N.G. Allen (ed.). Jet Cutting Tech. Mechan. Engng. Publ. Ltd., London (1994) 93-103

[15] R.A. Tikhomirov, V.B. Babanini, E.N. Pethukov, Highpressure jet cutting, ASME Press, New York (1992)

[16] J.O. Hallquist, LS-DYNA3D : Theoretical manual (Livermore Software Technology) (1997)

[17] W.F. Adler, Waterdrop impact modeling, Wear 186-187 (1995) 341-351

[18] LS-Dyna3D Course, Fluid/Structure coupling (DynalisChE-19-10-1998)

[19] D.J. Benson, Momentum advection on a staggered mesh, J. Comp. Phys. 100 (1992)

[20] M.C. Rochester, J.H. Brunton, High-speed impact of liquid jets on solids, 1st Int. Symp. on Jet Cutting Technology (Coventry 1972) 